\title{
EXPLORING CHALLENGES WITH DESIGNING AND DEVELOPING SHORE CONTROL CENTERS (SCC) FOR AUTONOMOUS SHIPS
}

\author{
H. Dybvik ${ }^{凶}$, E. Veitch and M. Steinert \\ Norwegian University of Science and Technology, Norway \\ $\square$ henrikke.dybvik@ntnu.no
}

\begin{abstract}
The concept of remotely operated, unmanned, and autonomous ships is creating increasing interest in the maritime domain, promising safety, increased efficiency and sustainability. Shore control centers (SCCs) have been proposed to operate such vessels and some industry projects are initiated. This paper aims at bringing knowledge about what a SCC is envisioned to be. It identifies and explores challenges related to designing and developing SCCs through semi-structured interviews with the research community and industry. We discuss tasks, functions and interactions between human and machine.
\end{abstract}

Keywords: autonomous ships, shore control center, design knowledge, information retrieval, early design phase

\section{Introduction}

Autonomous ships are creating increasing interest in the maritime domain, promising increased efficiency and sustainability. It is the goal of unmanned vessels to be at least as safe as manned vessels, in part because it moves seafarers away from potentially hazardous operations. An autonomous ship is a vessel with the possibility of operating on one or more Levels of Autonomy (LOA). This ranges from vessels with automated processes and decision support to remotely controlled and fully autonomous ships operating with or without seafarers onboard. Marine Autonomous Surface Ships (MASS) is the term suggested by the International Maritime Organization (IMO) to cover this broad range of automated and remotely controlled ships. Shore control centers (SCCs) have been proposed to operate such vessels (Levander, 2017; Lützhöft and Dekker, 2002; Rødseth et al., 2018) and to this date some industry projects have been initiated (MUNIN, 2015; Wilhelmsen, 2018).

Despite the recent interest in SCCs, there are currently no accepted guidelines for SCC design. As a result, some industry projects have approached the problem by replicating the ship's bridge onshore (see Figure. 1 for a prototype of an SCC and a traditional ship bridge). Research and systematic testing of alternative design approaches is lacking.

This paper aims at bringing forward knowledge about what an SCC is envisioned to be. It identifies and explores challenges related to designing and developing SCCs through semi-structured interviews with the research community and industry. We discuss expected tasks, functions, and interactions in the SCC and identify and describe associated design challenges.

Section 2 follows this introduction, providing background on why SCCs are needed and offers definitions for SCC and LOA. Section 3 covers methodology and Section 4 presents findings. Findings 
include how interviewees envisioned the SCC as well as what crucial interactions and design challenges might be encountered. Section 5 summarizes the work in a discussion; Section 6 presents a conclusion.

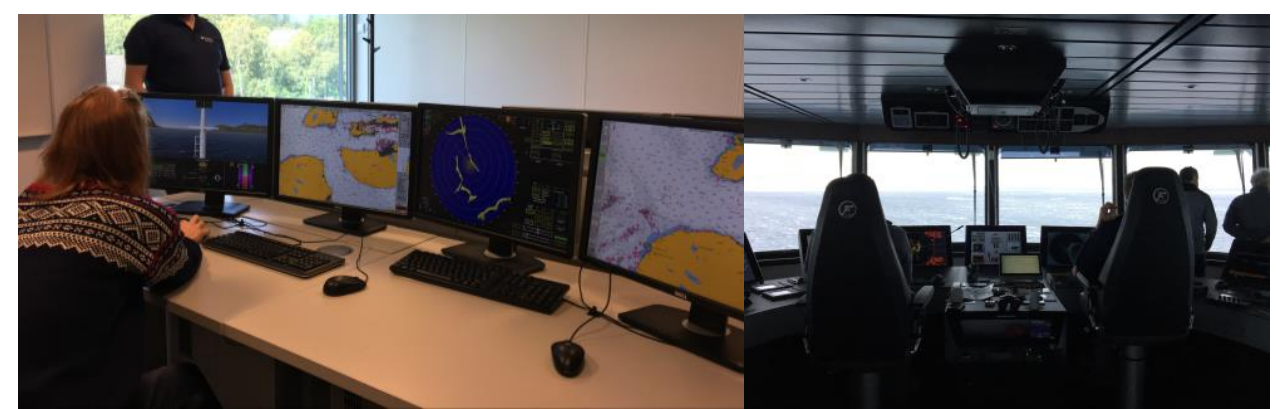

Figure 1. A prototype of an SCC (left) and a traditional ship bridge (right)

\section{Background}

\subsection{The need for SCCs}

The SCC is needed to monitor one or more autonomous ships remotely and to intervene in their navigation, if necessary. The word "autonomous" here does not describe a fully autonomous ship; rather, the moniker covers a range of autonomy levels onboard the ship's control system that stops before full autonomy. At Level 2 and Level 3 automation, according to the International Maritime Organization's (IMO) definition (IMO, 2018), the automation is not enough to allow the vessels to navigate without human supervision and the option to intervene via remote control is necessary. MUNIN (2015) represents the first study specifically undertaken to investigate design needs for SCCs. The project asserted that without a continuously manned SCC, design of an autonomous ship system would be very challenging. Leading design guidelines like the UK Code of Practice (Maritime UK, 2018) which to date only applies to MASS under $24 \mathrm{~m}$ in length, also highlight the importance of a "remote controller:" a person with whom responsibility of the vessel is assumed to lie and whose situational awareness and overview of the ship's mission is prerequisite for the operation's success. From the risk and reliability engineering perspective, too, have novel methods like Human-System Interaction in Autonomy (H-SIA) placed the SCC in a role of central importance as a mechanism for failure propagations of MASS scenarios (Ramos et al., 2019). Furthermore, autonomous and remotely operated ships operated by people onshore are expected to be safer, more efficient and cheaper to run (Ahvenjärvi, 2016; Levander, 2017), and reduce human error and workload (Lützhöft and Dekker, 2002). The risk of human errors due to fatigue is expected to reduce, leading to reduced risk of injuries to crew, ship, and cargo. Another advantage is the possibility to design these ships with a larger cargo capacity and reduced wind resistance. Certain features of today's ships, such as the deck house, the crew quarters, some ventilation, and sewage systems can be eliminated without crew onboard the ship. Despite advantages associated with introducing automation in the system, this also creates pathways to new technological and human errors, both of which have consequences (Lützhöft and Dekker, 2002).

Nevertheless, there is a general consensus that a fully autonomous vessel requiring no human input will likely not exist. Instead, a ship with highly automated control systems will require support from a human crew in the form of a Shore Control Center.

\subsection{SCC definitions}

While many definitions have been proffered by researchers and stakeholder organizations, there remains to be a widely accepted definition of the SCC. To date, even the terminology lacks unanimity, with the acronyms Shore Control Center (SCC), Remote Control Center (RCC), and Base Control Station (BCS) all appearing to describe what is by-and-large the same thing (Maritime UK, 2018; MUNIN, 2015; Rødseth et al., 2018; Rødseth and Nordahl, 2017). Remote Operating Center (ROC) has also been used in the research community (MTEC/ICMASS 2019, personal communication, 13-14. November 2019). In this work we use SCC. Rødseth and Nordahl (2017) defined the SCC simply as the "Owner's center for monitoring and control" and offered the following explanation of its function: 


\begin{abstract}
[The SCC] will be used partly as a backup in case the ship encounters unexpected events, partly to reduce the required complexity of on-board detection and control systems and partly to satisfy legal requirements that some human is in control of the ship.
\end{abstract}

This definition positions the SCC as a backup and legal requirement, rather than the central part of a wider monitoring and control system. MUNIN (2015) hypothesized the SCC in more specific terms, describing a control room in which each operator monitors and controls six vessels at a workstation containing six screens. Furthermore, requirements in the MUNIN set-up for operators to quickly respond to unexpected hazards raised questions about difficulties the operator may experience as "automation supervisor," known as "Out-Of-The-Loop" phenomenon (Kaber and Endsley, 1997). Ethnographic research examining the future skills of SCC operators points to unreasonably high demands on human capabilities given the current vision for the SCC (Lutzhoft et al., 2019).

\title{
2.3. Levels of Autonomy (LOA)
}

The concept of Levels of Autonomy, or levels of automation (LOAs) in human-machine interaction has been used to define which functions should be managed by the autonomy and which ones should be managed by the human operator (Musić and Hirche, 2017). For instance, Sheridan and Verplank (1978) defined 10 discreet LOAs, ranging from no autonomy to full autonomy. Endsley (1987) developed a 5-level LOA taxonomy regarding how much decision support would supplement human decision making during a cognitive task, ranging from manual support, to decision support, consensual artificial intelligence (AI), monitored AI and finally, full automation with no operator interaction.

In the context of autonomous ships and remotely operated vessels, several definitions of LOAs have been proposed. Autonomy Level 4, therefore, does not refer to full autonomy, but rather to "selfcontrolled function," whereby the system will execute the operation, but a human is able to override the action. IMO has identified four degrees of autonomy as a scoping exercise (IMO, 2018). Lloyd's Register (2016) has also defined 5 levels of autonomy in a design guideline for autonomous and remotely operated ships in terms of cyber access, ranging from complete human control (no cyber access; Level 1) to cyber access for autonomous/remote monitoring and control (onboard override not possible; Level 5). A 5-level definition has also been proposed by DNV-GL (DNV GL, 2018). It describes stepwise degrees in automatic control, where the Lloyd's definition is framed as discrete cyber accessibility levels. Rødseth et al. (2018) suggested building a framework based on SAE J3016 standard for autonomous cars and defined five basic types of autonomy in merchant vessels.

Generally, the LOA is expected to vary during the voyage according to level of risk and complexity. Accordingly, during low-autonomy sailing, human operators are expected to be highly engaged; during high-autonomy sailing, the operators are mainly in a supervising role.

\section{Method}

For an explorative study, a qualitative, adaptive approach using semi-structured interviews was chosen (Yin, 2017). First, the maritime ecosystem was mapped using the methodology behind a Customer Value Chain Analysis (Donaldson et al., 2006) to identify relevant interviewees. A total of 8 interviewees included Subject Matter Experts (SMEs) in the field of autonomous shipping and represented academic institutions, research organizations, and private sector companies in Norway. We used convenience sampling and snowball sampling through our own network. The interviewees were invited to take part via email.

Each interview lasted from 45-60 minutes and was conducted both in-person and via video call. Two researchers attended, one led the interview by asking questions and one wrote notes. Oral consent was obtained before beginning the interview. Beforehand, an interview protocol and an interview guide were made with predefined main questions. The interview began with basic questions about interviewees' backgrounds and current work. The main questions were selected to shed light on the study's primary investigation areas; namely, defining SCC, outlining future design challenges, defining operations, and critical human-system interactions. Towards the end we focused on the critical interactions as defined by the interviewee. Then we asked them to argue for the need for an SCC and give their impression of how the SCC business model would function. The questions were open-ended due to the explorative nature of the study, allowing the interviewee free train of thought 
and enabling unexpected insights. Flexibility around the questions enabled the interviewer to follow up on a specific train of thought and adapt the protocol with more specific questions. It also enabled the participants and interviewer to reach a mutual understanding in the instance where a question needed clarification or rephrasing, thus leading to more accurate data (Dörnyei, 2007).

The data was analyzed using cross case-synthesis (Eisenhardt, 1989; Yin, 2017), treating each interview as if it were a separate case.

\section{Findings}

\subsection{Envisioning the SCC - How is an SCC defined?}

This section describes how the interviewees communicated their vision for the SCC. Comparisons with and analogies to other forms of control centers were frequently mentioned by interviewees when envisioning the SCC. This was especially the case when describing physical infrastructure, data sources, and functions the SCC would perform.

The main purpose of SCCs is to provide the ability to take control of autonomous vessels from a remote location, especially as means to avoid critical situations, collisions, and allisions that are outside the capability of the automatic navigation algorithms. Thus, the display of ship-board sensory information was important. The operator's work inside the SCC may be characterized primarily as passive monitoring, similar to that of current-day Vessel Traffic Services (VTS). VTS operators are provided with information and have inputs enabling them to manipulate ships indirectly. The SCC will have access to similar data sources, such as Electronic Chart Display and Information Systems (ECDIS), VHF Marine Radio, ship schedule and voyage plan information, etc. The key difference between SCC and VTS operators is that the former has the ability to directly influence a ship's navigation as if they were in the bridge of the ship, only from afar.

When envisioning the physical SCC infrastructure, a set-up involving a combination of large screen displays and personal workstations was described to simultaneously provide a generalized "big picture" overview of the ships, along with the ability to search for and view more detailed information. Today, we need a clean setup. The layout of the room was important, and should be designed to minimize disturbances, provide good sightlines to the large-screen displays, and generally promote a sense of professionalism and ownership to the operators. Information should be shown according to priority to not overload the operator. Some descriptions revealed a resemblance to modern-day full-mission ship bridge simulators, with the key difference that it navigated a real ship and contained upgraded ECDIS with the possibility to view and adjust tracked trajectories with the means of a drag-and-drop feature. The argument was that current operators are used to being in a ship and therefore the more you can replicate a real ship, the better. Still, those who envisioned an immersive simulator environment conceded that this would not be necessary to get an acceptable situational awareness in an SCC.

An important distinction emerged between the functions of pure remote control and that of an SCC. Some industry projects are essentially copying the bridge and positioning it on-shore with a telecommunication link - an instance of remote control. On the contrary, the primary function of SCCs is the ability to adapt to different types of ships from one center. SCCs can be characterized as one center for multiple vessels of different types. Purely remote control "base stations," on the other, are one-to-one. This paper is not concerned with pure remote control.

A theme of transition from traditional to autonomous shipping emerged from the interview findings. SCC technology would not be disruptive; rather, it would be incremental by necessity of a highly regulated industry. This applied especially to competence needs, which is outlined in Section 4.3.5, whereby the SCC operators' qualifications may transition from those of experienced seafarers to those of younger operators with only virtual ship handling experience, who, while fully capable in their assigned role, would not hold the marine certifications that their predecessors once did. No one specified how long the transition would last, but several interviewees described a starting phase where the SCC would primarily be used for testing, verification, and approval. Later, the SCC would settle into overall transportation logistics management and might eventually coordinate to a higher degree with other available resources, like VTS. As such, the SCC they expected to see in the near future was distinctly different from the SCC they envisioned in the distant future. 


\subsection{Crucial interactions in a future SCC}

The human-machine interaction was said to be the most critical interaction in the future SCC. A "good connection" between the human and the automation system was needed. A prerequisite trust in the interaction system and automation was fundamental. The notion of trust includes buy-in from the industry, particularly from the shipowners' side in addition to trust from seafarers who will navigate ships alongside their unmanned counterparts. One interviewee said seafarers were sceptical of technologies that removed them from the location in which their work was done, since seafarers relied heavily upon their senses of sight, smell, sound, and proprioception. One example was given of an engine room operator's complaint, made during a participatory design process, about relocating a ship's engine control room far away from the engine room. The operator cited dissipation of vibration, sounds, and smells distant from the source as a potential hindrance to the operator's duties. Notwithstanding, the introduction of conditional monitoring software was accepted by the operator as making up for what may be lost by in-situ human monitoring, while having the benefit of placing the operator at a location more amenable to safe working conditions.

Researchers interviewed in this study were especially prone to citing human factor considerations as among the most important when designing the human-machine interactions and working environment in the SCC. Design of alarms was frequently mentioned as a method of communication that, if designed correctly, results in efficient detection of system prompts in order of priority.

\subsection{SCC design challenges}

\subsubsection{Handling the old model of seafaring}

Remote operation in the SCC was framed as a new paradigm in ship navigation. Yet, an old model of seafarers' traditional roles was acknowledged by many interviewees to dominate the emerging SCC mental model. Interviewees often described the imagined human-system interface in the SCC as the same that is found on-board contemporary ship bridges and VTS centers. They also commonly described the physical infrastructure of the SCC as a ship bridge moved to shore, albeit with incremental technology improvements. These assumptions appeared to bias some interviewees' reflections on SCC design challenges. For example, some described the challenges associated with moving the bridge to shore, confining the image of the SCC to that of ship's bridge with traditional bridge resource management. Some interviewees eschewed this assumption, instead framing the SCC as a new design space challenge. It appeared that copying the bridge onshore may not be the best solution and underscored the importance of separating the role of the SCC from that of the ship's bridge, including the competence needs of the operators occupying both spaces.

\subsubsection{Information display design}

With higher level technology and increasing automation there is an increasing access to a larger body of information, including different data sources and sensor information. Selecting which information to display and designing how it should be presented to provide the best possible overview of the situation is a challenge. One interviewee offered the model of top-down information search capability, in which the operator can effectively find specific information using a sequence of commands that narrows to the subsystem of interest. This top-down search should be complemented with a bottom-up information display model, in which specific information can quickly and effectively be placed within the larger context of the operation. An overarching task for the designer is to consider what information is displayed at what times, and how that information is presented in a way that does not overload operators.

\subsubsection{The human-automation handover design}

Designing the human-machine interface will be the most challenging part of SCC design, according to most interviewees. In particular, the handover; automation to human control will require special attention. During such a handover, some time will be required by the operator to gain situational awareness - time that is referred to as "getting in the loop". It is believed that with increasing autonomy the SCC will have less to with the voyage of the vessel, requiring the operator to monitor 
the vessel continuously. Should something disrupt the autonomy and require the operator to get into the loop, this adjustment time may be safety critical. Lowering the amount of time to get into the loop was one of the most important challenges cited. Currently, there is no test criteria regarding handover from automation to human, though one interviewee believed a systematic test approach was needed to approve SCCs. Results of studies from autonomous cars suggest handover times of approximately seven second (Gold et al., 2013), but there is a lack of experiments to test how long a handover takes in a ship from an SCC. Several interviewees in this study suggested that allowable ship takeover times may be longer than for cars, since ships have a relatively slow cruising speed.

The opposite direction of control handover, namely from human to automation, was considered less of a challenge. It was suggested that this process would be gradual and controlled, whereby the operator would hand over control, possibly in a stepwise process, and subsequently monitor the system for some time to ensure a successful handover.

\subsubsection{Communication}

Effective communication was highlighted by several interviewees as an essential component of the SCC design. SCC operators will have to communicate with VTS, port authorities, and other vessels. If it is a cargo vessel and it is part of a wider logistics system, there must also be communication with crane operators. One interviewee pointed out that modern regulations stipulate continuously being tuned in to VHF-radio onboard each ship, which would make communication chaotic when operating several vessels simultaneously. This was also mentioned by several other interviewees; having more than one communication channel would be an issue. They questioned how many vessels one can communicate with.

It was noted that limited bandwidth is a potential barrier to effective communication. This issue appears to escalate the farther offshore the vessel is located. Specifically, mobile phone broadband does not cover waters farther than coastal areas, and satellites are known to have latency issues and prohibitive cost associated with video and image transfer.

\subsubsection{Unknown skill requirements of SCC operators}

The skill requirement of the operator is largely unknown. Although there is currently research underway for SCC curricula, the emphasis has been mainly on hypothesizing a learning framework rather than on developing a tested and verified training program. Some interviewees suggested that opportunities for testing will present themselves with the first large-scale SCC applications and not before.

It was acknowledged that the skills necessary for SCC operators would be distinctly different from those of traditional seafarers. Still, it was conceded by most interviewees that during the initial stages of development, a trained seafarer with the required certificates in navigation and watchkeeping would have to be employed. Only when legal and regulatory adjustments are made for competence needs of SCCs will operators be trained in the core skills required for the job; namely, remote monitoring and control.

\subsubsection{Documentation and regulation}

The maritime industry is one of the most regulated industries. Work on-board ships follow strict protocols, procedures, and documentation processes. Ironically, some tasks are apparently conducted only for the sake of the documentation requirements themselves. Audits and inspections for safety management systems are in large part the cause for this inefficient process. For work to function seamlessly in the SCC, efficient documentation was therefore highlighted as an organizational design challenge. Instead of using documentation as a tool for passing inspection, other methods could be used for safety management. Video surveillance was suggested as a substitute for textual documentation, since it may provide the potential to record work procedures and automatically collect documentation electronically.

Regulations for SCCs are currently underdeveloped and have the potential to limit their value. One example was number of allowable ships monitored by one operator; if the number is below a certain threshold - or, worse, is only one - then the business case for remote monitoring and control would limit the intended function of the SCC. 


\subsubsection{How much automation is automation?}

There are several definitions of LOAs, none of which to our knowledge specifies exactly how much autonomy. Constrained autonomy was suggested as one way to clarify the boundaries of the automation system. It stems from the issue that maximizing intelligence may yield rigid boundaries in what the automation can and cannot do, resulting in abrupt handover exchanges with the operator. Constraining the automation such that self-limits its own functionality can circumvent this issue. If the automation detects, for instance, that the level of complexity in ship traffic has reached a certain level, rather than attempt to solve the situation alone, the system alerts the operators of an oncoming potential (and optional) handover. The operator is thus recruited to assess the situation and to judge whether human control is needed. This design has several additional benefits: improved communication of intent yields a higher degree of transparency and effectively stymies the Out-of-theLoop phenomenon. It is a drawback, too; namely, increased alarms. With good design implementation, though, alarms are thought to help normalize workload away from a more disjointed peak-trough pattern, potentially serving to improve vigilance over time.

\subsection{LOA task dependence}

All 8 interviewees agreed that tasks in the SCC will vary according to the LOA. A picture of adaptive automation emerged, wherein the LOA would adjust according to the voyage demands. When prompted to provide details for which tasks related to which LOA, responses were more abstract and ambiguous. Uncertainty around the definition of LOA appeared to be partly the cause for the lack of clear answers. Most interviewees cited "level 4 automation" as the highest attainable LOA on-board an autonomous ship. This referred not full autonomy, but rather to the self-controlled functionality whereby the ship system execute operation and a human is able to override it from the SCC. Interviewees were therefore referring to a LOA definition more in line with that of DNV-GL (DNV GL, 2018) than the IMO definitions that describe level 4 as fully autonomous.

\subsection{Challenging the assumption of if the SCC is needed}

Interviewees were asked whether the SCC was really needed and thus encouraged to challenge the underlying assumption of the study. All interviews agreed that the SCC was needed, but added several key exceptions.

Firstly, a distinction was made between small-scale autonomous vessels and their larger counterparts. In the former, the requirements for an SCC was questioned due to the shorter distances, proximity to shore, and overall lower perceived risk. For data collection vessels especially, like in the case of seabed surveys and hyperspectral imagery, it was noted that software can read the data better than humans at an SCC. For larger vessels, like cargo ships, some interviewees believed it will take many more years for the SCCs to develop due to the higher risk they posed to other ships.

Secondly, demand in traditional seafaring jobs and a shift in the seafarers' work content underscored the need for SCCs. Seafaring jobs were perceived to be less desirable among young people. The option to work from shore may appeal to some recruits. Moreover, more of the work on-board ships was perceived to be automated and screen-based, suggesting that the evolution to remote control is going to be incremental. The notion of "dirty, dull and dangerous" tasks, words which are often used to describe types of work that justify automation, was said by several interviewees as being prevalent during ship navigation.

One interviewee noted that should full autonomy be developed in the future, the SCC would not be needed.

\subsection{The business model of SCCs}

Several interviewees had given considerable thought to the business case of the SCC and shared their ideas with us; the majority, however, did not. The most immediate value proposition of automation ships appeared to be removal of humans from the vessel, saving cost and freeing space associated with hotel infrastructure and operation. Especially for smaller work vessels, removal of the hotel and amenities removes a considerable portion of the overall vessel infrastructure. An SCC is thus more 
cost-effective for smaller vessels. The value proposition is compounded with the ability of monitoring and controlling several vessels simultaneously.

It was noted that to date, traditional ship owners are not interested in autonomous ships. Rather, logistic and cargo distributions companies are showing the most interest. The shorter crossing and slower speeds of autonomous ships seem to suit areas where cargo distribution is needed, and predictable scheduling and broader logistics benefits outweigh the higher CAPEX of autonomous vessels. In some cases, the reduction of truck transportation is a major cost-saving element. The electrification of automation ships also meets public and stakeholder pressure on sustainability targets. From this point of view, the autonomous ships and the SCC represent a broad transportation system, distinct from the tradition model of shipping.

The need for volume was critical to the business case. One operator must be allowed to monitor more than one vessel simultaneously. The more vessels one operator (or one team of operators) could effectively manage, the better it was for profitability.

It was noted that insurers are general positive towards the notion of autonomous shipping. International P\&I clubs incur most costs due to payments associated with crew injuries.

\subsection{Sketching potential solutions}

Some interviewees challenged designers to frame the problem of SCC design with a higher degree of abstraction than simply moving the ship's bridge to shore. The process should start with the SCC and its unique requirements, not with how to solve the technical problems of a ship's remote control. Such a design strategy might result in simple and effective solutions.

Some specific solutions were offered by interviewees to address various components of the SCC design. For instance, regarding the limits of telecommunications (outlined in Section 4.3.4), one suggestion was to recreate digital imagery of objects detected in-situ, rather than continuously sending video and imagery to the SCC. In other words, the visual representation of detected obstacles, including their location in space, could appear as a simulation in the SCC, removing the need for expensive, limited, and delayed transfer of images.

Almost all interviewees highlighted the Out-of-the-Loop phenomenon as a major design challenge for SCCs. One interviewee suggested the "constrained autonomy" could be an effective mitigator (Section 4.3.7). The constrained autonomy model also improves transparency and may normalize workload and fit well into a larger framework of adaptive automation.

Finally, experimentation was highlighted as a key to development of SCCs. Currently many critical elements - like the number of vessels one operator can manage, for instance - are hypothesized. Systematic testing and verification are necessary for approval and acceptance.

\section{Discussion}

Many interviewees compared the SCC to control centers found in other domains and industries. VTS and air traffic controller (ATC) were most frequently mentioned. Power plants, space centers, centralized fish farming, train line monitoring, and autonomous cars were also mentioned as applications of remote control from which SCCs could adopt design elements.

In the ship navigation, rigid hierarchical roles define bridge management. One interviewee challenged the assumption that SCC operators will follow the same order of command, suggesting that the SCC may be a venue better suited for flatter organizational structure. In any case, SCC resource management will be an area for design consideration.

The concept of "ship sense" was occasionally discussed by interviewees, most often in the context of a set of distinct human qualities - be that on the bridge, engine room, or elsewhere - that operators rely upon to do their job well. These human qualities, explicitly mentioned by interviewees, included the senses of smell, touch, sight, and a sense of "embodiment" which the authors understand to mean proprioception. Interviewees expressed that removing the operators from the location of their work would effectively hamper ship sense, tacit knowledge required for operators to navigate ships effectively. While interviewees generally agreed on this definition, there emerged two school of thought regarding its role in the SCC. Some were in favour simulating ship sense in the SCC, with examples such as integrated motion beds, vibrating seats, and smell alarms. Others were not in favour 
of this concept, stating that a combination of condition monitoring sensors and alarms were more reliable than the human senses, and that distance from the sources of risk may enable safer and more effective decision making. Ahvenjärvi (2016) asks if the best of the human element will be lost by autonomous ship technology. We extend this question to how human factors can be applied in SCC design research to best adapt to human sensemaking, especially in safety critical situations.

All interviewees expressed a high degree of trust in current automation technology. This is interesting because it's remarkably different from other interview-based studies about human factors needs in the transition to automation shipping, which revealed trust issues as a major concern (Mallam et al., 2019). The difference may be because of the shift in interviewees' focus; interviewer envisioning operators in a monitoring and control role may place more trust in the autonomous systems compared to a third party, like another ship.

It was accepted that one operator (or one SCC team unit) is required to operate several vessels for it to be a viable business case. The questions of how many vessels remains unanswered. Many interviewees cited studies that showed that this number was 6 to 10 vessels. This refers to the MUNIN project, in which 6 vessels was set as the upper limit; however, MUNIN (2015) states that that this number is hypothesized and as of today still not verified. In any case, setting a fixed upper limit may be problematic, since manoeuvres like docking require more attention than open-water transiting. The number will also be dependent on additional variables, such as location, voyage, and LOA. The maximum number of ships one operator can manage is therefore likely to encompass a wide range rather than a single number.

Many interviewees were mentally fixated with the old model of seafaring. Such a design fixation (Moreno et al., 2015) might be a problem both during user testing and during design work, and must be overcome.

\section{Conclusion}

The concept of remotely operated, unmanned, and autonomous ships is creating increasing interest in the maritime domain, promising safety, increased efficiency and sustainability. SCCs have been proposed to operate such vessels and some industry projects are initiated. This paper aims at bringing knowledge about what a SCC is envisioned to be. It identifies and explores challenges related to designing and developing SCCs through semi-structured interviews with the research community and industry. We discuss tasks, functions and interactions between the human operation and autonomous ship control system. There are many unknowns and challenges regarding design, development and operations of SCCs. These include, but are not limited to, the human-automation handover, management of the transition from traditional shipping, design fixation, information display design, communications, and number of vessels an operator can handle. Currently, many critical elements like the number of vessels one operator or one team can manage - are hypothesized and represent important research tasks. Experimentation and systematic testing may unlock solutions to SCC design challenges and will be necessary for regulatory approval and user acceptance. SCCs represent an emerging complex socio-technical system and introduces many new risks and unknown unknowns. Considering the consequences of failure stemming from poor implementation, design research is needed to address the challenges associated with incorporating the human in the SCC as operator of autonomous ships.

\section{Acknowledgement}

The authors would like to thank interviewees for their time and contribution. This research is supported by strategic funds from the department for Mechanical and Industrial Engineering (MTP) at NTNU.

\section{References}

Ahvenjärvi, S. (2016), “The Human Element and Autonomous Ships”, TransNav Int. J. Mar. Navig. Saf. Sea Transp., Vol. 10. https://doi.org/10.12716/1001.10.03.18

DNV GL. (2018), Class Guideline DNVGL-CG-0264 Autonomous and remotely operated ships.

Donaldson, K.M., Ishii, K. and Sheppard, S.D. (2006), "Customer Value Chain Analysis", Res. Eng. Des., Vol. 16, pp. 174-183. https://doi.org/10.1007/s00163-006-0012-8 
Dörnyei, Z. (2007), Research methods in applied linguistics: Quantitative, qualitative, and mixed methodologies, Oxford University Press, Oxford.

Eisenhardt, K.M. (1989), "Building Theories from Case Study Research”, Acad. Manage. Rev., Vol. 14, pp. 532550. https://doi.org/10.2307/258557

Endsley, M.R. (1987), "The Application of Human Factors to the Development of Expert Systems for Advanced Cockpits", Proc. Hum. Factors Soc. Annu. Meet., Vol. 31, pp. 1388-1392. https://doi.org/10.1177/15419 3128703101219

Gold, C. et al. (2013), “Take over!' How long does it take to get the driver back into the loop? Proc. Hum. Factors Ergon", Soc. Annu. Meet., Vol. 57, pp. 1938-1942. https://doi.org/10.1177/1541931213571433

IMO (2018), IMO takes first steps to address autonomous ships [WWW Document]. URL http://www.imo.org/en /MediaCentre/PressBriefings/Pages/08-MSC-99-MASS-scoping.aspx (accessed 11.24.19).

Kaber, D.B. and Endsley, M.R. (1997), "Out-of-the-loop performance problems and the use of intermediate levels of automation for improved control system functioning and safety”, Process Saf. Prog., Vol. 16, pp. 126-131. https://doi.org/10.1002/prs.680160304

Levander, O. (2017), “Autonomous ships on the high seas”, IEEE Spectr., Vol. 54, pp. 26-31, https://doi.org/10. 1109/MSPEC.2017.7833502

Lloyd's Register (2016), Cyber safe for marine [WWW Document]. Lloyds Regist. URL https://www.lr.org/en/ cyber-safe-for-marine/ (accessed 11.22.19).

Lutzhoft, M. et al. (2019), "Human-centred maritime autonomy - An ethnography of the future", J. Phys. Conf. Ser., Vol. 1357, p. 012032. https://doi.org/10.1088/1742-6596/1357/1/012032

Lützhöft, M.H. and Dekker, S.W.A. (2002), "On Your Watch: Automation on the Bridge”, J. Navig., Vol. 55, pp. 83-96. https://doi.org/10.1017/S0373463301001588

Mallam, S.C., Nazir, S. and Sharma, A. (2019), "The human element in future Maritime Operations-perceived impact of autonomous shipping”, Ergonomics, pp. 1-12.

Maritime UK (2018), Maritime Autonomous Surface Ships - UK Code of Practice | Maritime UK [WWW Document]. URL: https://www.maritimeuk.org/media-centre/publications/maritime-autonomous-surfaceships-uk-code-practice/ (accessed 11.25.19).

Moreno, D.P. et al. (2015), “A step beyond to overcome design fixation: a design-by-analogy approach", In: Design Computing and Cognition '14, Springer. pp. 607-624.

MUNIN (2015), D8.8: Final Report: Shore Control Centre - FP7 GA-No 314286 | All MUNIN Deliverables [WWW Document]. URL: http://www.unmanned-ship.org/munin/news-information/downloads-information -material/munin-deliverables/ (accessed 11.25.19).

Musić, S. and Hirche, S. (2017), "Control sharing in human-robot team interaction", Annu. Rev. Control, Vol. 44, pp. 342-354. https://doi.org/10.1016/j.arcontrol.2017.09.017

Ramos, M. et al. (2019), "Human-system concurrent task analysis for maritime autonomous surface ship operation and safety", Reliab. Eng. Syst. Saf., p. 106697.

Rødseth, ØJ and Nordahl, H. (2017), "Definitions for autonomous merchant ships", Presented at the Norwegian Forum for Unmanned Ships, Version, pp. 2017-10.

Rødseth, ØJ, Nordahl, H. and Hoem, А. (2018), Characterization of Autonomy in Merchant Ships, in: 2018 OCEANS - MTS/IEEE Kobe Techno-Oceans (OTO). Presented at the 2018 OCEANS - MTS/IEEE Kobe Techno-Oceans (OTO), pp. 1-7. https://doi.org/10.1109/OCEANSKOBE.2018.8559061

Sheridan, T.B. and Verplank, W.L. (1978), Human and computer control of undersea teleoperators, Massachusetts Inst of Tech Cambridge Man-Machine Systems Lab.

Wilhelmsen. (2018), Wilhelmsen and KONGSBERG establish world's first autonomous shipping company [WWW Document]. Wilhelmsen. URL: https://www.wilhelmsen.com/media-news-and-events/pressreleases/2018/wilhelmsen-and-kongsberg-establish-worlds-first-autonomous-shipping-company/ (accessed 11.22.19).

Yin, R.K. (2017), Case study research and applications: Design and methods, Sage publications. 